\title{
The future of Chemoecology: part II
}

\author{
Günther Raspotnig ${ }^{1}\left[\right.$ ] Marko Rohlfs ${ }^{2}(\mathbb{D}$
}

Published online: 27 February 2019

(c) Springer Nature Switzerland AG 2019

As announced recently, Michael Heethoff has resigned from his job as Editor-in-Chief of Chemoecology (Heethoff 2018). We, Günther Raspotnig (University of Graz, Austria) and Marko Rohlfs (University of Bremen, Germany) took over as Editors-in-Chief in January 2019.

We have a long-standing experience as Associate Editors of Chemoecology, strong bonds with the journal, and are convinced that chemoecological research still provides fundamental and innovative mechanistic concepts of how species interactions affect populations and communities, and how chemical signals have been shaped by natural selection. In this respect, we will continue Chemoecology as a solid, pioneering, basic research-oriented journal. Yet, we feel to reconsider what matters in modern chemoecology and propose an update of the Aim and Scopes of Chemoecology. The results of our thoughts will soon appear on the journal webpage.

In particular, we will be supporting initiatives for Special Issues meant to serve as a catalyst for emerging common themes. We, therefore, encourage individual researchers and teams to propose and discuss topics for Special Issues, and to act as guest editors.

Along with this update of Aims and Scopes, we have been able to assemble a new and fresh team of Associated Editors covering a wide range of chemoecological topics. We are very much looking forward to a fruitful collaboration with:

Stefan Dötterl, Paris Lodron University of Salzburg, Austria.

Patrick Fink, Helmholtz Centre for Environmental Research, Magdeburg, Germany.

Günther Raspotnig

guenther.raspotnig@uni-graz.at

Marko Rohlfs

rohlfs1@uni-bremen.de

Institute of Biology, University of Graz, Graz, Austria

2 Population and Evolutionary Ecology Group, University of Bremen, Bremen, Germany
Catrin Günther, The New Zealand Institute for Plant \& Food Research, Auckland, New Zealand.

Almuth Hammerbacher, University of Pretoria, Department of Zoology and Entomology, South Africa.

Zain Seyd, University of Kentucky, Department of Entomology, United States of America.

With these changes, we believe to provide an excellent platform for critically discussing, promoting and disseminating the vast and fascinating diversity of current chemoecological research.

\section{Reference}

Heethoff M (2018) The future of chemoecology. Chemoecology 28:75

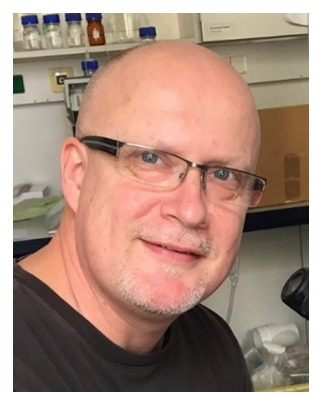

Günther Raspotnig works as a Senior Lecturer at the Institute of Biology, Graz, Austria. He has published more than 60 papers on the Chemical Ecology of Arthropoda, thereby focusing on the evolutionary history of secretion chemistry in exocrine systems and phylogenetic chemosystematics, respectively.

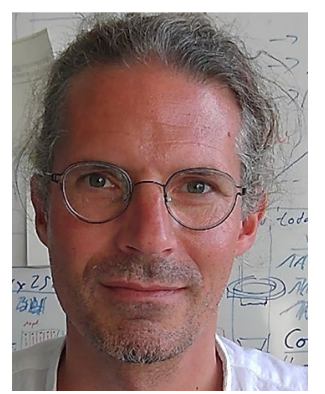

Marko Rohlfs , Population and Evolutionary Ecology Group, Bremen, Germany. My research interest focuses on the evolutionary chemical ecology of insectfungus interactions. 